ARTICLE

DOI: $10.1038 / \mathrm{s} 41467-018-06757-2$

\title{
Predictive multiphase evolution in Al-containing high-entropy alloys
}

\author{
L.J. Santodonato ${ }^{1,3}$, P.K. Liaw ${ }^{2}$, R.R. Unocic (10 ${ }^{1}$, H. Bei (1) ${ }^{1}$ \& J.R. Morris (1) ${ }^{1,2}$
}

The ability to predict and understand phases in high-entropy alloys (HEAs) is still being debated, and primarily true predictive capabilities derive from the known thermodynamics of materials. The present work demonstrates that prior work using high-throughput first-principles calculations may be further utilized to provide direct insight into the temperature- and composition-dependent phase evolution in HEAs, particularly Al-containing HEAs with a strengthening multiphase microstructure. Using a simple model with parameters derived from first-principles calculations, we reproduce the major features associated with Alcontaining phases, demonstrating a generalizable approach for exploring potential phase evolution where little experimental data exists. Neutron scattering, in situ microscopy, and calorimetry measurements suggest that our high-throughput Monte Carlo technique captures both qualitative and quantitative features for both intermetallic phase formation and microstructure evolution at lower temperatures. This study provides a simple approach to guide HEA development, including ordered multi-phase HEAs, which may prove valuable for structural applications.

\footnotetext{
${ }^{1}$ Oak Ridge National Laboratory, Oak Ridge, TN 37831, USA. ${ }^{2}$ Department of Materials Science and Engineering, The University of Tennessee, Knoxville, TN 37996, USA. ${ }^{3}$ Present address: Advanced Research Systems, Macungie, PA 18018, USA. These authors contributed equally: L. J. Santodonato, J. R. Morris. Correspondence and requests for materials should be addressed to J.R.M. (email: morrisj@ornl.gov)
} 
T he fundamental nature of structural order-disorder transitions in binary alloys was explained in the early twentieth century, in large part due to the work of Bragg and Williams, by considering only the nearest-neighbor interaction energies and the configurational entropy of atoms populating a fixed lattice ${ }^{1}$. The present work uses a similar approach to model ordering and related cooling transformations, which often occur in multi-element alloys, such as high-entropy alloys (HEAs) ${ }^{2-15}$. The present examination of HEA cooling transformations originated in the context of the entropy hypothesis that disordered solid-solution phases will be stabilized in HEAs, due to their high configurational entropy, relative to the competing phases. A recent comprehensive review ${ }^{4}$ has established that enthalpy plays a critical role in determining which compositions form single solid-solution phases with no long-range configurational order. Troparevsky et al. have demonstrated that predicting single-phase compounds may largely be achieved by considering the enthalpy gain of forming ordered phases from each potential binary combination $^{7,16}$. This procedure may be done strictly from highthroughput first-principle calculations, without input from experiments, and largely through a remarkably simple "enthalpy matrix" derived from a large set of calculations.

Clearly, the above approach is one step in a broader fundamental problem: one would like to know not only that a particular composition will show multiple phases, but further what phases would be present at any given temperature, ideally with minimal input from experimental work. To address this challenge, we consider the $\mathrm{Al}_{x} \mathrm{TM}$ system, where $\mathrm{TM}$ represents combinations of $3 d$ transition-metal (TM) elements, such as $\mathrm{CoCrFeNi}$. This system is chosen due to the broad number of studies within the HEA literature ${ }^{2,10,17-21}$, and due to the fact that for sufficient $\mathrm{Al}$, there are a series of phase transformations, including the formation of partially ordered aluminides in a body-centered-cubic (BCC) solid-solution matrix ${ }^{10,22-24}$. By controlling the composition and microstructure through thermal processing associated with the phase transformations, this system provides a wide range of potential compounds and associated microstructures. The strong ordering tendencies of $\mathrm{Al}$ with most of the TMs makes the ordering transition of primary importance. As reported in the early literature, some HEAs have roomtemperature microstructures of ordered and disordered phase mixtures ${ }^{2,17}$. The $\mathrm{Al}_{x} \mathrm{CoCrFeNi}$ alloy forms coherent mixtures of B2 and BCC phases at room temperature ${ }^{10,17,21,25-27}$, particularly when the $\mathrm{Al}$ contents are large $(x>0.875)^{28}$. The six-component $\mathrm{AlCoCrCuFeNi}$ alloy has a three-phase FCC/BCC/B2 microstructures under most processing conditions, with the facecentered-cubic (FCC) phase being primarily a Cu-based bystander phase that minimally affects the other transformations ${ }^{17}$.

Below, we present both calculations and experiments that are consistent with these main transformations in $\mathrm{Al}_{x} \mathrm{CoCrFeNi}$, for $1<x<2$ : on cooling from high temperatures (near melting), the high-temperature BCC solid solution transforms into a partially ordered B2 phase. Upon further cooling, the partially ordered B2 phase transforms into a phase mixture comprised of disordered BCC (primarily $\mathrm{Co}$ and $\mathrm{Fe}$ ) plus a B2 phase (primarily composed of Ni-Co-Al). This two-step transformation is illustrated using Monte Carlo simulations (Fig. 1).

\section{Results}

Monte Carlo model for multi-component transformations. The present Monte Carlo simulations are implemented, using a lattice model, where the atoms are constrained to reside on a fixed lattice -in the present case, a BCC lattice. This model is based on the experimental knowledge of the $\mathrm{Al}_{x} \mathrm{CoCrFeNi}$ phases, and represents the only input from experiment. The simulated cooling

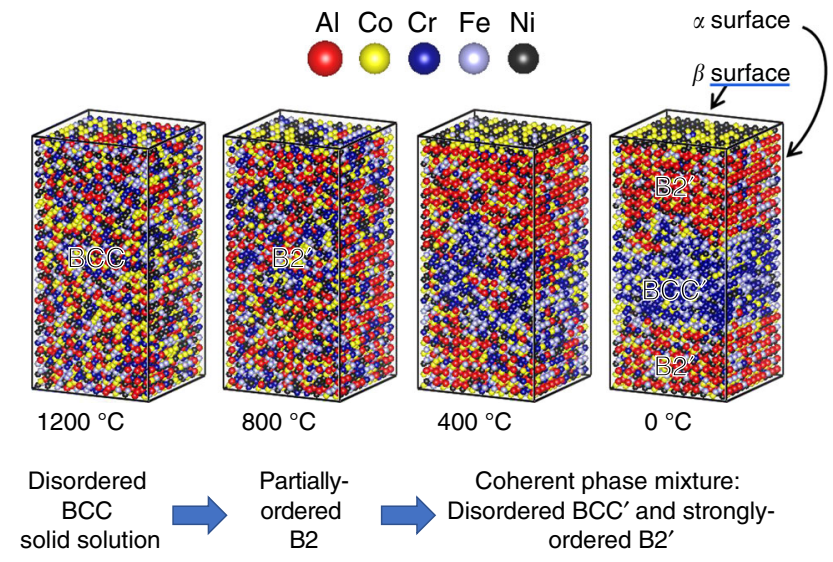

Fig. 1 Monte Carlo simulations of a two-step cooling transformation in the $\mathrm{AICoCrFeNi} \mathrm{HEA}$. The atomic distributions for a series of different temperatures are illustrated, using supercells, composed of $12 \times 12 \times 24$ cubic unit cells, where each unit cell contains one $\alpha$ and one $\beta$ site. The supercells are "cut" to highlight configurational ordering, such that the side surfaces contain Al-rich $\alpha$ sites, and the top surface contains Al-poor $\beta$ sites. Based upon the element-specific long-range order parameters (Fig. 3c), we find that the high-temperature phase is a disordered solid solution, which transitions to a partially ordered phase during cooling to $800^{\circ} \mathrm{C}$. Upon further cooling, the partially ordered phase transforms into a mixture of disordered $\mathrm{Cr}$-Fe-enriched $\mathrm{BCC}$ and strongly ordered $\mathrm{Al}-\mathrm{Co}-\mathrm{Ni}$ enriched B2 phases

transformations involve only redistributions of the atomic population, as an approximation to the behavior of real alloys undergoing sublattice ordering and coherent phase separation. This lattice constraint is too restrictive to address all types of HEA transformations, or to provide high-accuracy simulations: it neglects both the possibility of changing the underlying lattice, and also ignores potential vibrational entropy effects associated with small displacements from the average position ${ }^{29}$. However, the lattice-constrained simulations correctly predict the major trends in some well-known HEAs ${ }^{2,10,17}$, especially in their as-cast forms, and provide a quantitative framework for understanding the tendencies to both short- and long-range chemical ordering. The shortcomings are addressed in the Discussion section below.

For simplicity, the interactions are assumed to be nearneighbor only, and solely dependent upon the composition of the near-neighbor sites. The values of the binary interaction energies, $v_{i j}$, are derived from the enthalpy matrix, $H_{i j}$, associated with the enthalpy change on ordering from a solid solution, which were recently assembled and applied to the problem of predicting HEA compositions likely to form single-phase solid solutions ${ }^{7}$. The relationship between the $H_{i j}$ and $v_{i j}$ is given by

$$
v_{i j}=\frac{H_{i j}}{z}
$$

where $z$ is the number of the first nearest neighbors in the anticipated HEA structure. This assumption is motivated mainly by the convenience of directly applying the existing $H_{i j}$ matrix to the calculations. The Monte Carlo moves are the simple exchanges of neighboring atoms, with usual Metropolis acceptance probabilities. The details are specified in the Methods section below.

The values of $v_{i j}$ are given in Table 1 , for the simulations presented, as well as some related components. These values are reasonable, in light of prior experimental observations. First, the interactions between Co-Cr-Fe-Mn-Ni are all small (magnitudes 


\begin{tabular}{|c|c|c|c|c|c|c|c|}
\hline Elements & Al & Co & $\mathrm{Cr}$ & $\mathrm{Cu}$ & $\mathbf{F e}$ & Mn & $\mathbf{N i}$ \\
\hline $\begin{array}{l}\mathrm{Al} \\
\mathrm{Co} \\
\mathrm{Cr} \\
\mathrm{Cu} \\
\mathrm{Fe} \\
\mathrm{Mn} \\
\mathrm{Ni}\end{array}$ & $\begin{array}{l}0 \\
-7.58 \\
-1.66 \\
-1.80 \\
-4.44 \\
-3.35 \\
-8.15\end{array}$ & $\begin{array}{l}-7.58 \\
0 \\
0.06 \\
0.65 \\
-0.72 \\
-0.23 \\
-0.25\end{array}$ & $\begin{array}{l}-1.66 \\
0.06 \\
0 \\
1.30 \\
-0.10 \\
-1.32 \\
-0.36\end{array}$ & $\begin{array}{l}-1.80 \\
0.65 \\
1.30 \\
0 \\
0.78 \\
0.35 \\
-0.07\end{array}$ & $\begin{array}{l}-4.44 \\
-0.72 \\
-0.10 \\
0.78 \\
0 \\
0.11 \\
-1.17\end{array}$ & $\begin{array}{l}-3.35 \\
-0.23 \\
-1.32 \\
0.35 \\
0.11 \\
0 \\
-1.39\end{array}$ & $\begin{array}{l}-8.15 \\
-0.25 \\
-0.36 \\
-0.07 \\
-1.17 \\
-1.39 \\
0\end{array}$ \\
\hline
\end{tabular}
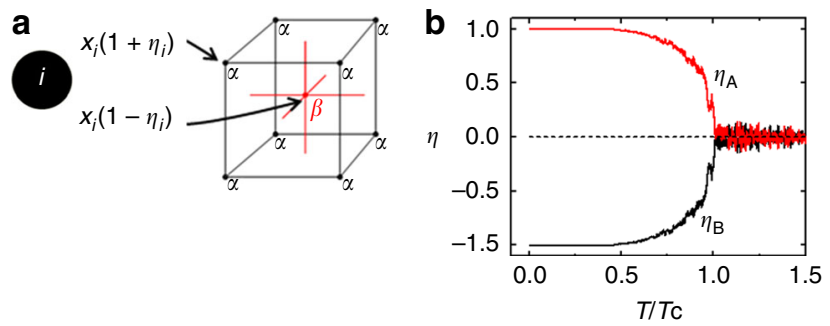

Fig. 2 Configurational order parameters. a An illustration shows how multiple elements, each with a mole fraction, $x_{i}$, fill the interpenetrating lattices, $\alpha$ and $\beta$, according to a set of order parameters, $\eta_{i}$. $\mathbf{l}$ In the case of a binary alloy, there is only one independent order parameter, $\eta=\eta_{\mathrm{A}}=-\eta_{\mathrm{B}}$, which is normally plotted over a range of 0 to 1 , and as a function of the reduced temperature, $T / T_{C}$, where $T_{\mathrm{C}}$ is the ordering temperature. Here both $\eta_{\mathrm{A}}$ and $\eta_{\mathrm{B}}$, are plotted, over the range of -1 to 1 , as will be necessary for HEAs with four or more principal elements. The plots contain some statistical noise because they were generated from Monte Carlo simulations

$<2 \mathrm{~kJ} / \mathrm{mol}$ ), indicating the weak interactions in the single-phase forming "Cantor" alloy of $\mathrm{CrCrFeMnNi}{ }^{3}$. Second, the addition of Al shows a favorable interaction with every other component, with particular strong interactions with $\mathrm{Ni}$ and Co. This trend immediately suggests the formation of a Ni-Co-Al phase at low temperatures, as has been observed ${ }^{17,21,25}$. Roughly speaking, the $\mathrm{Co}$ and $\mathrm{Ni}$ interactions are reasonably similar, and our results presented below demonstrate that $\mathrm{Co}$ and $\mathrm{Ni}$ have similar behaviors. Finally, we note that with the exception of weakly attractive interactions with $\mathrm{Ni}$ and $\mathrm{Al}, \mathrm{Cu}$ has a positive interaction with all other components. Thus, we may expect that $\mathrm{Cu}$ will tend to segregate away from the others. This trend has been observed experimentally, and is supported by simulations ${ }^{10,17,30,31}$.

Below, two distinct approaches are used to characterize the development of ordered compounds in the simulated work. The nearest-neighbor pair correlations, $P_{i j}$, are defined as the average fraction of neighbors to the type- $i$ atoms that are type- $j$ atoms. The $P_{i j}$ are normalized such that $\Sigma_{j} P_{i j}=1$, and $P_{i j}$ give the probability that any given nearest neighbor of the element $i$ is of the type $j$. In the case of a randomly mixed single phase, the $P_{i j}$ simply correspond to the fractional concentrations of the potential neighbors, such that $P_{i j}=x_{j}$. Deviations from the $P_{i j}=x_{j}$ random behavior reveal element-specific ordering, segregation, and phase separation, which are critical issues in the development of HEAs.

The long-range ordering behavior of a single-phase HEA may be expressed, employing a set of parameters, $\eta_{i}$, for $i=1$ to $n$ elements. The $\mathrm{B} 2 \mathrm{(CsCl}$ ) structure, relevant for the formation of $\mathrm{NiAl}$ and other aluminides, may be represented using two interpenetrating sublattices on the BCC lattice, $\alpha$ and $\beta$, with equal numbers of sites, $a^{\alpha}=a^{\beta}=1 / 2$ (Fig. 2a). The fractional occupancy of element $i$ on sublattice $\alpha$ is denoted as $y_{i}^{\alpha}$, and the associated order parameters are given by

$$
\eta_{i}=\frac{y_{i}^{\alpha}-y_{i}^{\beta}}{y_{i}^{\alpha}+y_{i}^{\beta}}
$$

The $\eta_{i}$ values fall within the range of -1 to 1 , and are related to experimentally observed quantities, such as neutron and X-ray diffraction patterns ${ }^{10}$, as shown below. The case of $\eta_{i} \approx 0$ indicates that the element, $i$, is found on both sublattices in equal proportions. When $\eta_{i}$ approaches positive or negative 1, however, the element is found exclusively on the $\alpha$ or $\beta$ sublattice, respectively. The opposite values represent the different possible symmetry-breaking arrangements. For alloys with $n>2$ elements, the use of multiple order parameters allows an unambiguous description of the atomic-ordering behavior. Each sublattice contains half of the total number of sites, $N$. Vacancies are not considered.

Despite its simplicity, the present model allows us to understand HEA-ordering behaviors in greater depth than the standard classifications based upon binary prototype alloys, such as the B2ordered $\mathrm{CsCl}$. Although it is correct and useful to classify the HEAs in this fashion, additional information is needed because there are many distinct ways to achieve a given ordering type for compositions of $n>2$ major elements. The problem may be solved, using sets of element-specific parameters, such as the sublattice occupancies, which have been used in the study of the MoNbTaW alloy ${ }^{32}$, or with order parameters, $\eta_{i}$, used here and in earlier work on the $\mathrm{Al}_{1.3} \mathrm{CoCrCuFeNi}$ alloy ${ }^{10}$. Indeed, our work here is similar in spirit to studies on the MoNbTaW alloys ${ }^{32,33}$, though we use a simpler Monte Carlo scheme (utilizing only first neighbors and without the use of molecular dynamics simulations).

Simulated order-disorder transitions and transformations in HEAs. All simulated systems are disordered at high temperatures but form an ordered structure as the system is cooled. The progression of structures is shown for $x=1$ in Fig. 1. To demonstrate this feature more rigorously, and to quantify the development of order, plots of $\eta_{i}$ versus temperature from Monte Carlo simulations are shown in Fig. 3, for $\mathrm{Al}_{x} \mathrm{CoCrFeNi}$, with $\mathrm{Al}$ compositions ranging from $x=1$ to $x=2$. One significant feature of the simulations is that the ordering onset, i.e., where the HEA is cooled below the ordering temperature, $T_{\mathrm{c}}$, involves the strong ordering of at least two, but not necessarily all, of the $n>4 \mathrm{TM}$ elements. The dominant behavior on cooling is that $\mathrm{Al}$ segregates to one sublattice, with $\mathrm{Ni}$ and Co primarily occupying the other sublattice. All behaviors are quantitatively reproduced on both heating and cooling simulations, indicating that the results are essentially in equilibrium. The effect of the $\mathrm{Al}$ content is 
a

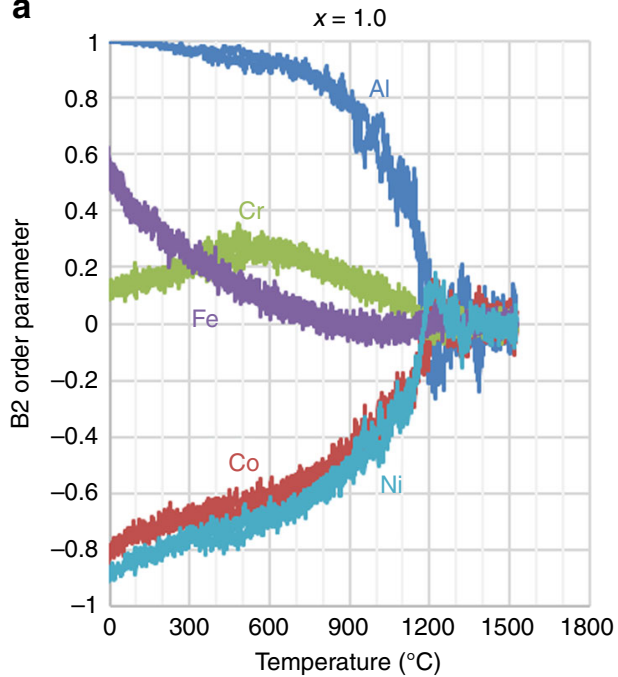

C

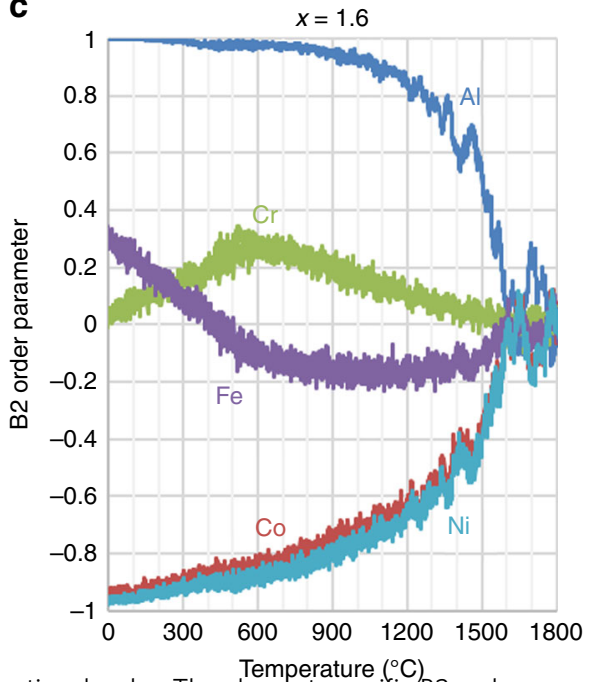

b

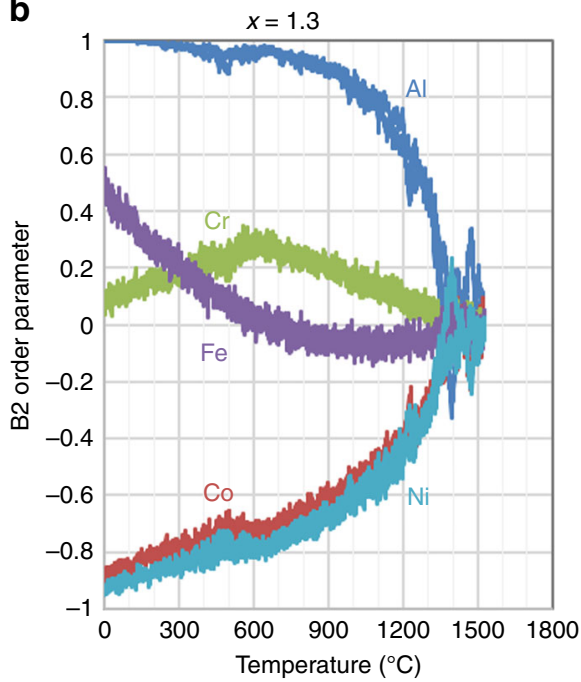

d

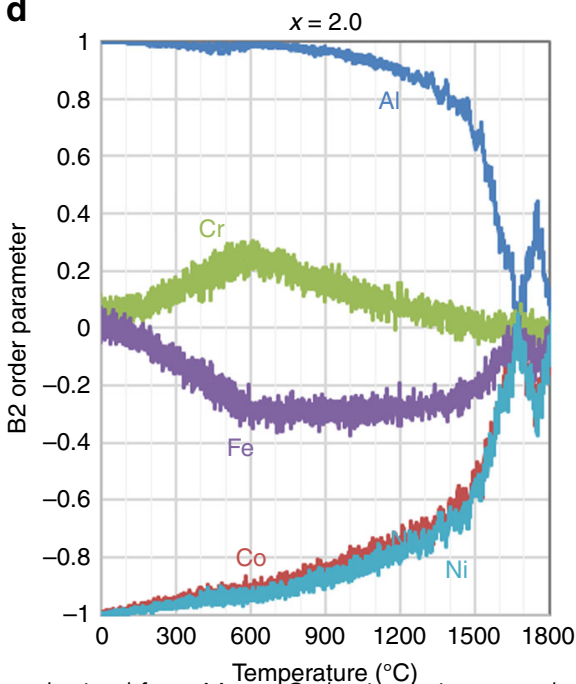

Fig. 3 Simulated configurational order. The element-specific B2-order parameters obtained from Monte Carlo simulations are plotted for the $\mathrm{Al}_{x} \mathrm{CoC} r \mathrm{FeNi}$ family of alloys. A comparison of four different aluminium contents, $\mathbf{a} x=1.0, \mathbf{b} x=1.3, \mathbf{c} x=1.6$, and $\mathbf{d} x=2.0$, shows that the order parameters all tend toward zero above a sufficiently high temperature (i.e., the ordering-onset temperature), which increases with increasing Al content. More importantly, the two distinct behaviors of the strongly ordering ( $\mathrm{Al}, \mathrm{Co}$, and $\mathrm{Ni}$ ) and disordered $(\mathrm{Cr}$ and $\mathrm{Fe}$ ) groups of elements, which are seen in all the plots, are crucial to understanding the two-step cooling transformations of the present alloys

demonstrated by comparing the $\mathrm{Al}_{2} \mathrm{CoCrFeNi}$ and $\mathrm{AlCoCrFeNi}$ HEA systems. In the higher-Al-content $\mathrm{Al}_{2} \mathrm{CoCrFeNi}$ alloy, $T_{\mathrm{c}}$ is above the experimental melting point, such that the alloy never becomes disordered in the solid phase. Instead, the alloy is partially ordered at the melting temperature. In the lower-Al composition, AlCoCrFeNi, $T_{c}$ occurs in the solid phase, near $1200^{\circ} \mathrm{C}$. A more complete description of the $\mathrm{B} 2$-ordering trends are discussed below. The fraction of Al-Al pairs is low at all temperatures, indicating that even above the B2-transformation temperature, the $\mathrm{Al}$ atoms are primarily pairing with the TMs. Interestingly, Fig. S1 shows that as the Al content increases, the fraction of Al-Al pairs decreases, opposite of what would normally be expected for a disordered material. This trend is due to the stronger tendency to form a B2 phase with increasing $\mathrm{Al}$ content. Thus, even in the high temperature, disordered BCC phase, there is significant short-range order around the $\mathrm{Al}$ atoms, in contrast to descriptions where the $\mathrm{Al}$ is assumed to be randomly organized ${ }^{34}$.

In order to examine the development of B2 order experimentally, in situ neutron scattering studies were conducted from room temperature all the way to the molten phase, using an aerodynamic levitator ${ }^{35,36}$, in a similar fashion to the previous work ${ }^{10}$. The new diffraction patterns of both the $\mathrm{Al}_{2} \mathrm{CoCrFeNi}$ (Fig. 4a) and AlCoCrFeNi (Fig. 4b) demonstrate that the B2/BCC structural evolution proceeds entirely by the rearrangement of atoms on a common BCC lattice, producing a single family of diffraction peaks (i.e., all peaks are indexed to a common lattice parameter) throughout the temperature-dependent phase evolution. These neutron diffraction results highlight the remarkable tendency of the presently studied family of HEAs to form simple structures, even when they undergo ordering and phase separation.

Within the observed family of diffraction peaks, we distinguish between fundamental (common to both the disordered BCC and ordered B2 structures) and superstructure reflections (exclusive to the $\mathrm{B} 2$ structure). Both types coexist in ratios that vary with temperature. In the $\mathrm{Al}_{2} \mathrm{CoCrFeNi}$ alloy, the superstructure peaks persist all the way to the melt (Fig. 4a), while in the equimolar $\mathrm{AlCoCrFeNi}$ alloy, they are observable only at $1000^{\circ} \mathrm{C}$ and below (Fig. 4b). The experimental results in Fig. 4 are consistent with 


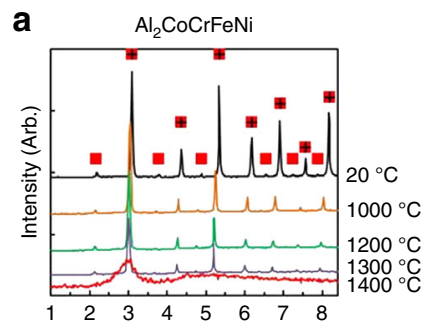

$Q\left(\AA^{-1}\right)$

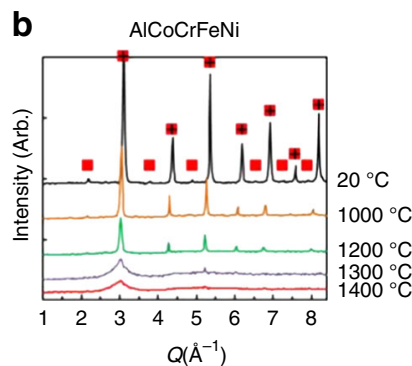

Peak types: + BCC fundamental

B2 superstructure

C

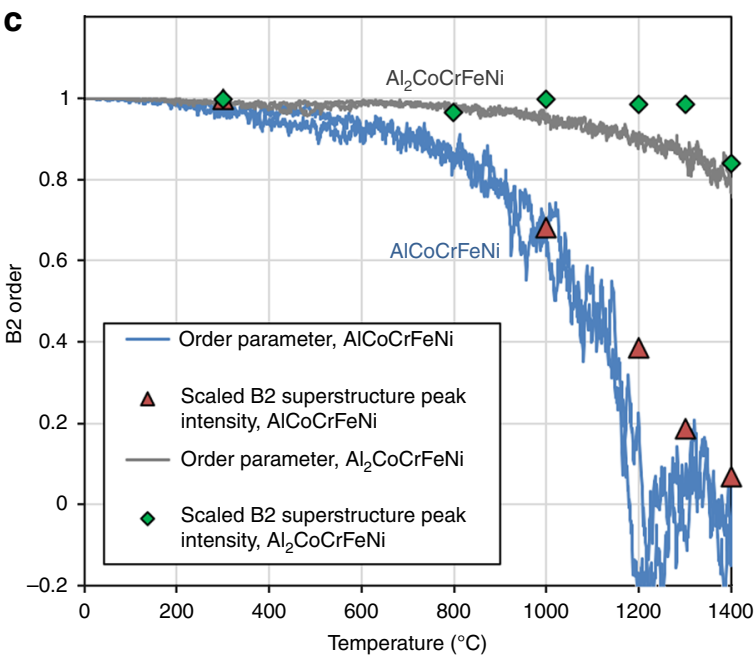

Fig. 4 Neutron diffraction patterns from in situ levitated heating studies, compared with simulations. a The plots of intensity versus scattering vector magnitude, $\mathrm{Q}$, show that the $\mathrm{Al}_{2} \mathrm{CoCrFeNi}$ alloy produces one family of peaks corresponding to the $\mathrm{BCC} / \mathrm{B} 2$ structure. The $\mathrm{B} 2$ superstructure peaks persist until the alloy melts near $1400^{\circ} \mathrm{C}$, indicating that the alloy never reaches the disordered state in the solid phase. $\mathbf{b}$ The $\mathrm{AlCoCrFeNi}$ alloy has a similar BCC/B2 structure. However, the superstructure signal becomes weak during heating, and is not observable at $1200^{\circ} \mathrm{C}$ and above, indicating that the alloy may undergo an order-disorder transition. $\mathbf{c} A$ comparison of the $B 2$ superstructure diffraction peak intensity for $Q$ near $2.2 \AA^{-1}$, scaled by the value at $300^{\circ} \mathrm{C}$, with the $\mathrm{B} 2$-order parameter, $\eta_{\mathrm{Al}}$, from simulations. Note that the highest experimental temperatures shown here are close to the melting transformation. One general feature of all compositions is the peak shift due to thermal expansion

the formation of a partially ordered B2 phase, which occurs directly from the melt in the high-Al-content $\mathrm{Al}_{2} \mathrm{CoCrFeNi}$ alloy, and through an order-disorder transition in the $\mathrm{AlCoCrFeNi}$ alloy. To compare with simulations, Fig. $4 \mathrm{c}$ shows the superlattice peak intensity, $S(Q)$, for the peak near $2.2 \AA^{-1}$ for both systems, scaled by their value at $T=300{ }^{\circ} \mathrm{C}$. The $\mathrm{B} 2$-order parameter, $\eta_{\mathrm{Al}}$, from simulations is also shown. The comparison between the diffraction studies and the simulation is strikingly close: both show the loss of the $\mathrm{B} 2$ order for $\mathrm{AlCoCrFeNi}$ near $1400{ }^{\circ} \mathrm{C}$, while the $\mathrm{B} 2$ phase remains strongly ordered at that temperature for $\mathrm{Al}_{2} \mathrm{CoCrFeNi}$. This trend demonstrates that our simulations quantitatively capture the development of B2 order in these systems.

Temperature-dependent ordering within the BCC solid solution. The simulations show a rapid change in short-range chemical order for all compositions, at temperatures well below the
B2-ordering temperature, demonstrated for $\mathrm{Al}_{2} \mathrm{CoCrFeNi}$ in Fig. 5. While composition changes and phase transformations are expected during cooling, we observe a rapid change over a narrow temperature range, where the weakly ordered $\mathrm{Cr} / \mathrm{Fe}$ atoms appear to precipitate from the $\mathrm{B} 2$ phase into a disordered solid-solution phase, simultaneous with the enhanced ordering behavior of the B2 phase. This trend is exhibited in Fig. 1 for the $x=1 \mathrm{com}-$ pound, where a weakly ordered system at $800^{\circ} \mathrm{C}$ clearly is twophase at $400^{\circ} \mathrm{C}$. The sharpness of this change in behavior for $x=$ 2 is evident in Fig. 5, showing the distribution of different pair types. The $P_{i j}$ continuously change with respect to temperature throughout the transformation, although there is an abrupt change in the trend near $600^{\circ} \mathrm{C}$. This is particularly true for the $\mathrm{Fe}-\mathrm{Fe}$ and $\mathrm{Cr}-\mathrm{Cr}$ correlations (Fig. 5a) and other $\mathrm{Cr}$ correlations (Fig. 5d). Cr and Fe are identified as disordered because the $P_{\mathrm{Cr}-\mathrm{Cr}}$ and $P_{\mathrm{Fe}-\mathrm{Fe}}$ values are close to the random-mixing value of $x_{\mathrm{Cr}}=$ $x_{\mathrm{Fe}}=1 / 6$ at temperatures above $700{ }^{\circ} \mathrm{C}$ (Fig. 5a), and at all temperatures shown, $P_{\mathrm{Cr}-\mathrm{Fe}} \approx P_{\mathrm{Cr}-\mathrm{Cr}}$ and $P_{\mathrm{Fe}-\mathrm{Fe}} \approx P_{\mathrm{Fe}-\mathrm{Cr}}$, indicating that $\mathrm{Cr}$ and $\mathrm{Fe}$ continue be to be closely mixed even as these quantities change rapidly below $600^{\circ} \mathrm{C}$. For $\mathrm{Cr}$, above this temperature, the number of $\mathrm{Co}, \mathrm{Cr}, \mathrm{Fe}$, and $\mathrm{Ni}$ pairs are approximately equal (Fig. 5d); below this temperature, the number of $\mathrm{Cr}$ $\mathrm{Cr}$ and $\mathrm{Cr}-\mathrm{Fe}$ pairs rapidly increases, while the number of other Cr-TM pairs rapidly drops. The dramatic increase in both the $P_{\mathrm{Cr}-\mathrm{Cr}}$ and $P_{\mathrm{Fe}-\mathrm{Fe}}$ (Fig. 5a) at temperatures below $600{ }^{\circ} \mathrm{C}$ is an indication of phase separation, occurring during cooling. The $\mathrm{Al}$, $\mathrm{Co}$, and $\mathrm{Ni}$ elements are identified as ordered because their selfpair probabilities all tend toward zero during cooling (Fig. 5a), and strongly paired with $\mathrm{Ni}$ and $\mathrm{Co}$ (Fig. 5b). This feature is consistent with the order-parameter analysis in Fig. 3, showing strong ordering of $\mathrm{Ni} / \mathrm{Co}$ with $\mathrm{Al}$ for all temperatures below $\sim 1600{ }^{\circ} \mathrm{C}$ (the ordering onset occurs at $1850{ }^{\circ} \mathrm{C}$ ) for the $x=2$ composition. This transition behavior is further shown in the pairs of $\mathrm{Al}, \mathrm{Fe}$, and $\mathrm{Cr}$ pairs, in Fig. $5 \mathrm{~b}-\mathrm{d}$. Figure $5 \mathrm{~b}$ shows that above $600{ }^{\circ} \mathrm{C}$, the fraction of $\mathrm{Fe}-\mathrm{Al}$ pairs is nearly constant. However, cooling below this temperature, the number of pairs drop off dramatically.

As discussed below, the transition near $600^{\circ} \mathrm{C}$ is seen for all compositions between $x=1$ and 2 , and is roughly temperatureindependent. Similar results are observed for all compositions, as presented in the Supplementary Figures 1 and 2 showing the evolution of pair fractions for $\mathrm{Al}$ and $\mathrm{Cr}$ for all compositions. Supplementary Figure S2 also shows that the ratio $P_{\mathrm{Cr}-\mathrm{Ni}} / P_{\mathrm{Cr}-\mathrm{Cr}}$ changes sharply near $600{ }^{\circ} \mathrm{C}$ for all systems, and serves as a strong indicator for this change.

In situ scanning transmission electron microscopy (STEM) and differential scanning calorimetry (DSC) experiments support these observations. The DSC work on AlCoCrFeNi $(x=1)$ shows a peak near $575^{\circ} \mathrm{C}$, in the range of the simulated value of $565 \pm 30^{\circ} \mathrm{C}$ (see the Supplementary Figure 3). In situ STEM was conducted, while heating an $\mathrm{Al}_{1.3} \mathrm{CoCrCuFeNi}$ alloy from room temperature to $700^{\circ} \mathrm{C}$. This alloy was previously studied using in situ neutron and $\mathrm{X}$-ray diffraction, from room temperature all the way to the melt ${ }^{10}$. As noted earlier and in the literature previously ${ }^{10}$, the $\mathrm{Cu}$ phase separates, and does not significantly affect the transitions in the remaining matrix. Only subtle changes were observed in the diffraction patterns between room temperature and $700{ }^{\circ} \mathrm{C}$, which were attributed to the temperature evolution of the highly coherent microstructure dominated by dual BCC/B2 phases. Because the dual phases have nearly the same crystal periodicity, it was recognized that major microstructural transformation may be responsible for the rather small changes in the diffraction signals. The present STEM study provides the direct experimental observation needed to verify these microstructural transformations. 
a

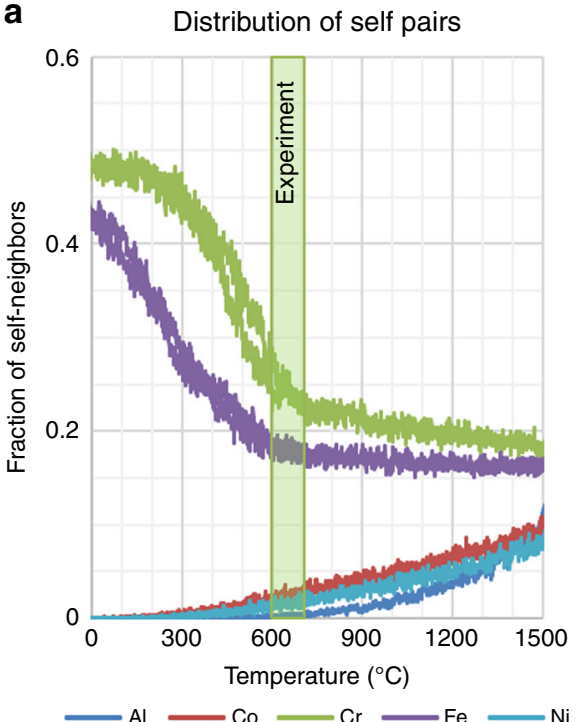

C

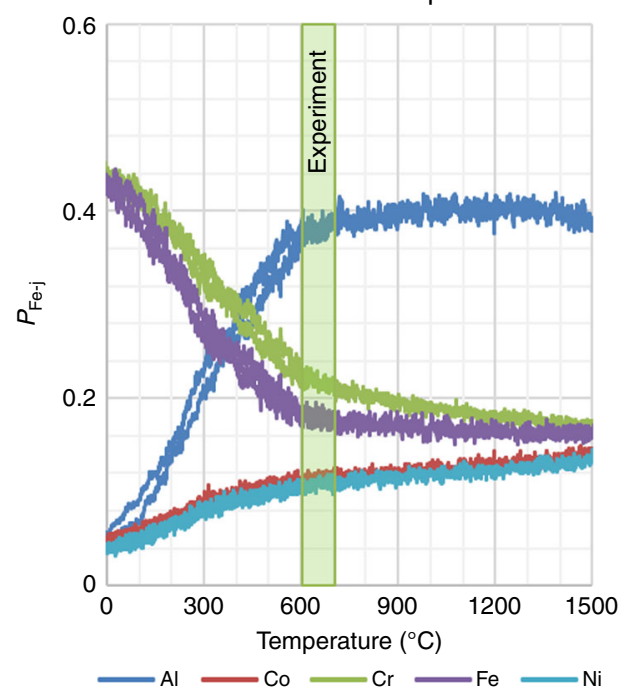

b

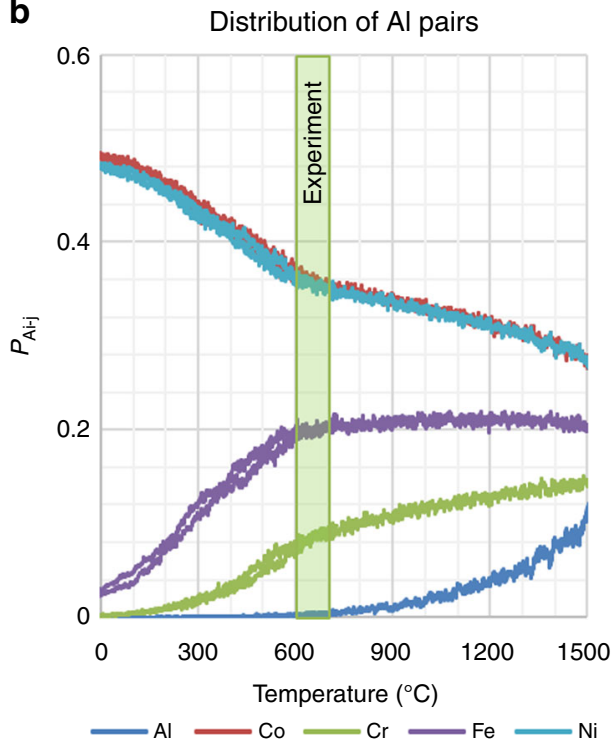

d

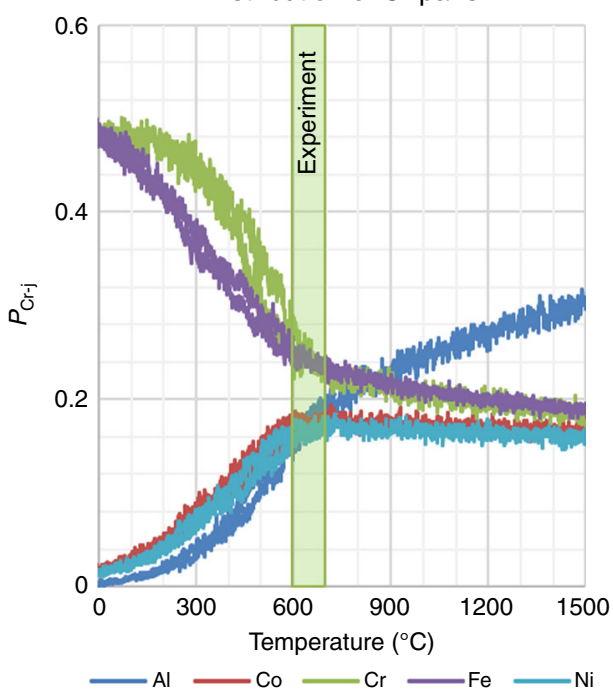

Fig. 5 Nearest-neighbor-pair correlations reveal transformations in $\mathrm{Al}_{2} \mathrm{CoCrFeNi}$. The temperature range where microscopy shows a rapid change in microstructure $\left(600-700^{\circ} \mathrm{C}\right.$; Fig. 6$)$ is highlighted by the shaded box in parts a-d. a The self-pair probabilities, $P_{i i}$, reveal two groups of elements, the strongly ordering $\mathrm{Al}, \mathrm{Co}$, and $\mathrm{Ni}$, with vanishing self-neighbor probability at low temperatures that indicates strong ordering, and the disordered/ segregating $\mathrm{Cr}$ and Fe. The strongly ordering and disordered/segregating groups play key roles in the first and second steps, respectively, of the cooling transformation. $\mathbf{b}$ The $P_{\mathrm{Al}-j}$ indicate that $\mathrm{Co}$ and $\mathrm{Ni}$ are the strongly preferred neighbors of Al. While the fraction of Fe neighbors is nearly constant above $600^{\circ} \mathrm{C}$, this trend drops off sharply below this temperature. c The $P_{\mathrm{Fe}-j}$ show a distinct change near $600^{\circ} \mathrm{C}$, consistent with segregation from the Al-rich matrix. d The $P_{\mathrm{Cr}-\mathrm{j}}$ show a similar trend as the $P_{\mathrm{Fe}-j \mathrm{j}}$ except that the $\mathrm{Cr}$-Al-pair probability is lower

A plate-like microstructure, as found in many $\mathrm{Al}_{x} \mathrm{CoCrCuFeNi}$ alloys ${ }^{10,17,21,26}$, is observed in the room-temperature $\mathrm{Al}_{1.3} \mathrm{CoCr}$ $\mathrm{CuFeNi}$ micrograph (Fig. 6a), which is color-coded according to abundances of $\mathrm{Al}$ (red), $\mathrm{Cr}$ (green), Fe (blue), and Ni (magenta) detected from the energy dispersive spectrometry (EDS). The Co and $\mathrm{Cu}$ EDS signals are suppressed to more clearly highlight the differences between the matrix, having a reddish appearance, and the blue/green plates, which are enriched in $\mathrm{Cr}$ and $\mathrm{Fe}$ (Fig. 6a). The $\mathrm{Cr}$-Fe-enriched plates begin to decompose upon heating to $600{ }^{\circ} \mathrm{C}$, such that the $\mathrm{Fe}$ and $\mathrm{Cr}$ migrate to separate regions within plates, as shown by the nonuniform color (Fig. 6b). Further heating to $700{ }^{\circ} \mathrm{C}$ causes a complete breakdown of the plate-like morphology, such that the globular Cr-rich regions are dispersed throughout the matrix, and the Fe appears to have dissolved into the matrix (Fig. 6c). Schematic illustrations (Fig. 6d-f) are shown below the micrographs (Fig. $6 \mathrm{a}-\mathrm{c}$ ) to give a simplified version of the observed microstructure transformation.

Our Monte Carlo simulations exhibit very similar behaviors. The temperature range over which the morphology is observed to change is presented as green bars in Fig. 5. As we see, this feature closely corresponds to the changes observed in the simulated short-range order. The reduced ordering of $\mathrm{Fe}$, compared to $\mathrm{Cr}$, is seen both in the experiment and in the pair behavior of Fig. 5a, where the $\mathrm{Fe}-\mathrm{Fe}$ pairs are essentially random above $700{ }^{\circ} \mathrm{C}$, while $\mathrm{Cr}$-Cr pairs retain significant order to much higher temperatures. Figure $5 \mathrm{~b}$ also shows that there is a sharp change in the $\mathrm{Fe}-\mathrm{Al}$ pairs over this temperature range, while the $\mathrm{Cr}-\mathrm{Al}$ pair presents a much more gradual change. This feature is consistent with the observed rapid change in behavior of Fe near $600{ }^{\circ} \mathrm{C}$ (Fig. 6b, e), and the more gradual 
a
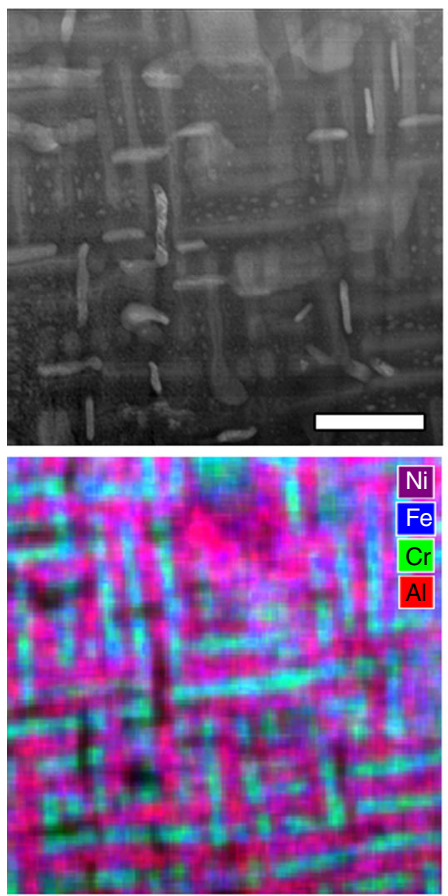

d

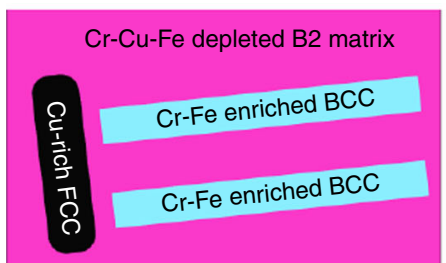

b
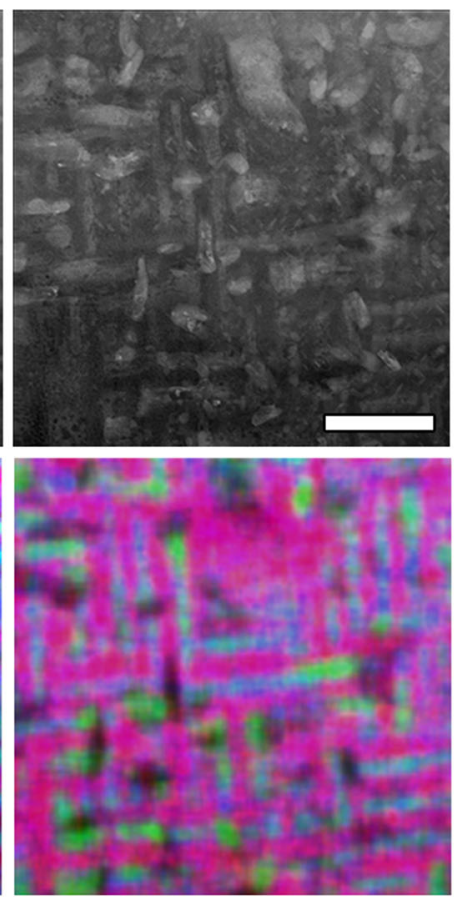

e

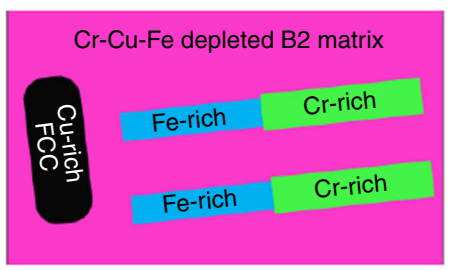

C
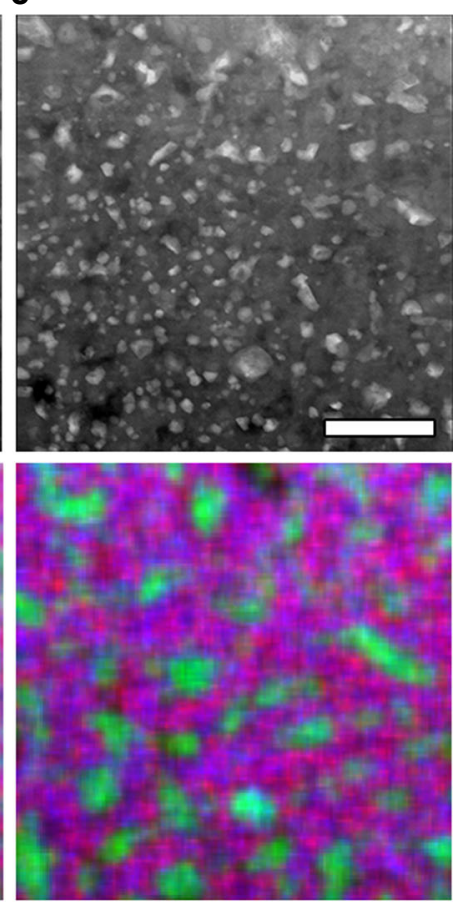

$\mathbf{f}$

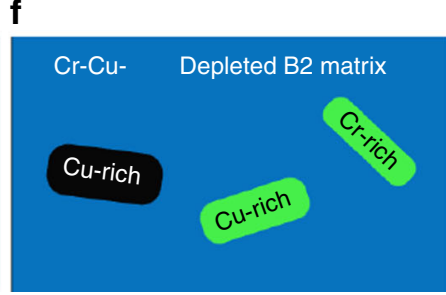

Fig. 6 In situ STEM/EDS demonstrate rapid changes in the microstructure between 600 and $700^{\circ} \mathrm{C}$. Representative ADF STEM images and corresponding EDS Maps ( $\mathrm{Ni}, \mathrm{Fe}, \mathrm{Cr}$, and $\mathrm{Al}$ ) of the $\mathrm{Al}_{1.3} \mathrm{CoCrCuFeNi}$ microstructure. a Initial room-temperature microstructure shows an $\mathrm{Al}-\mathrm{Ni}$-enriched matrix with $\mathrm{Cr}$ Fe-enriched plates. $\mathbf{b}$ Heat treatment at $600^{\circ} \mathrm{C}$ causes separation of $\mathrm{Cr}$ and Fe within the plates. $\mathbf{c}$ Heat treatment at $700^{\circ} \mathrm{C}$ breaks down the coherent microstructure. The observed microstructures are represented schematically in $\mathbf{d}$ room temperature, e $600{ }^{\circ} \mathrm{C}$, and f $700^{\circ} \mathrm{C}$. Scale bars are $500 \mathrm{~nm}$

change in the Cr-rich regions on heating to $700{ }^{\circ} \mathrm{C}$ (Fig. $6 \mathrm{c}, \mathrm{f}$ ). We note that the length scales in our simulations are dramatically different than those on the microscope images, and that these limited length scales may prevent a full description of the behavior. The Supplementary Figures 1 and 2 show that the simulations reveal only a weak dependence of this transformation with the $\mathrm{Al}$ content.

We observe the following trends for $\mathrm{Al}_{x} \mathrm{CoCrFeNi}$, for the range $1<x<2$, summarized in Fig. 7 : there is a strongly $x$ dependent $\mathrm{B} 2$-ordering temperature, ranging in the simulations from approximately $1220^{\circ} \mathrm{C}$ for $x=1$ to $1850^{\circ} \mathrm{C}$ for $x=2$. For $x=1$, this value is close to the temperature where $\mathrm{B} 2$ superlattice peaks are first observed (Fig. $4 \mathrm{~b}$ ). These compounds melt near $1300{ }^{\circ} \mathrm{C}$ (Fig. 4a, b). Thus, for $x=2$, the prediction is that the compound will have strong B2 order on solidification, consistent with our scattering experiments (Fig. 4a). We predict that the system will have weak B2 order on solidification for most compositions in this range. We further predict a weakly $x$ dependent temperature where the $\mathrm{B} 2$ and BCC solid solutions become strongly separated. This unexpectedly sharp transformation occurs very closely to an observed change in the microstructure, using in situ microscopy during heating (Fig. 6b, c), and at a temperature very close to a peak observed through calorimetry for $x=1$ (Supplementary Figure S3). The observed transition is quite similar to the simulations, with the B2 phase being primarily $\mathrm{Ni}, \mathrm{Co}$, and $\mathrm{Al}$ (Figs. 2 and $6 \mathrm{~b}$ ), and the disordered phase being primarily $\mathrm{Fe}$ and $\mathrm{Cr}$ (Fig. 4a, c).

Even though the pathway to phase separation and microstructure development in HEAs may begin with sublattice ordering and coherent phase separation, the equilibrium microstructures sometimes contain phases that are structurally unrelated to the primary solid-solution structures. For example, recent studies using the calculation of phase diagrams (CALPHAD) on the $\mathrm{Al}_{x} \mathrm{CoCrFeNi}$ alloys have predicted the formation of sigma phases, which experimentally appear only after hundreds of hours aging at temperatures in the range of $700-1000^{\circ} \mathrm{C}^{22,23}$. The as-cast alloys show the simpler microstructures, containing FCC, BCC, and their ordered derivatives ${ }^{28}$. The present simulations focus on the observed BCC-based metastable microstructures, which form during the casting process, and even in many heat-treated alloys, in order to explore the HEA temperaturecomposition space to identify regions, where interesting and potentially useful transformations occur. The transformations proceed in such a way that the configurational entropy of the overall atomic population decreases during cooling, as expected, although retaining significant disorder in the lattice.

\section{Discussion}

The results presented here represent a remarkably simple theoretical description of multiple transitions occurring in 


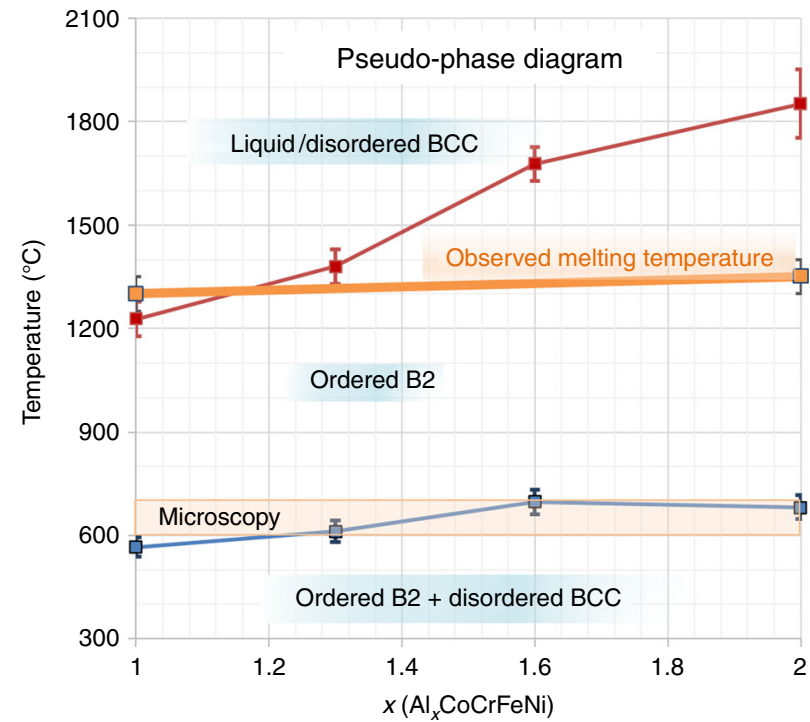

Fig. 7 Schematic phase diagram for solid phases of $\mathrm{Al}_{x} \mathrm{CoCrFeNi}$ in the range from $1<x<2$. This diagram captures the simulated trends in $\mathrm{B} 2-$ ordering temperature with $\mathrm{Al}$ content (red data points) and the temperature at which the primarily $\mathrm{Cr}-\mathrm{Fe} \mathrm{BCC}$ phase develops (blue data points). Note that above $\sim 1300^{\circ} \mathrm{C}$, the solid is observed to melt (orange line); thus, for higher Al content, the melt will crystallize into the B2 phase, as observed in scattering experiments presented in Fig. 4. The lower transition is also marked by the $600-700^{\circ} \mathrm{C}$ range of temperatures where in situ microscopy demonstrates a change in the microstructure, as shown in Fig. 6

Al-containing HEAs. The Monte Carlo results presented here have no parameters from experiment, derived instead from the enthalpy matrix presented in ref. ${ }^{7}$, which is based upon highthroughput first-principle calculations. The advantage of the Monte Carlo simulations is that they build in the enthalpies associated with forming ordered phases, while simultaneously capturing the large amounts of disorder (and associated entropy) that occur in each of the phases. These high-throughput simulations are based entirely on nearest-neighbor interactions on a rigid lattice, in the same fashion as the early studies on binary systems ${ }^{1}$, and successfully reproduce major features of the phase evolution observed in Al-containing HEAs, including the B2ordering behavior at high temperatures, and the $\mathrm{B} 2 / \mathrm{BCC}$ separation near $600{ }^{\circ} \mathrm{C}$.

While we expect that interactions beyond near-neighbor pairs would be significant, and furthermore that other thermodynamic considerations (particularly vibrational entropy effects ${ }^{29,37-40}$ ) that are presently ignored in our model here, the results demonstrate both qualitative and reasonably quantitative comparisons to experiment: scattering provides a key test for B2 ordering (Fig. 4), while the predicted B2/BCC separation near $600{ }^{\circ} \mathrm{C}$ (Fig. 5) is supported by in situ microscopy experiments (Fig. 6), and DSC (Supplementary Figure 3). The current approach suggests a broader approach to rapid exploration of phase evolution, where little experimental data exist. The present strategy directly explores the chemical ordering in these "highentropy" and related complex concentrated alloys, providing an important complement to other important techniques, such as CALPHAD in the quest for developing next-generation materials.

\section{Methods}

Neutron scattering. Neutrons, having no electric charge, are a highly penetrating probe for studying the structural and dynamic properties of materials ${ }^{41,42}$. Neutron scattering techniques include diffraction, which gives information on the longrange order of crystalline materials, and pair distribution function analysis, which gives information on the local atomic order.

Neutron scattering is well-suited for the present work, because the technique is sensitive to the ordering of the multiple TM elements within HEAs, and the experiments may be conducted using special environments for in situ heating and melting. In the present work, neutron scattering studies were performed at the Oak Ridge National Laboratory, Spallation Neutron Source, using the Nanoscaleordered Materials Diffractometer ${ }^{43}$. The experiments were conducted over a wide temperature range, from room temperature to $1600^{\circ} \mathrm{C}$, using an aerodynamic levitator ${ }^{10,35,36}$. The levitator provides a containerless environment, in which small samples ( 2-mm-diameter spheres) are suspended above a conical nozzle with flowing argon gas, and heated with a $250 \mathrm{~W} \mathrm{CO}_{2}$ laser operating at a wavelength of $10.6 \mu \mathrm{m}$. Therefore, the levitator ensures that the phase transformations are not influenced by interactions between the sample and container, allowing in situ neutron studies of the intrinsic transformations. Typical neutron measurement times were $15 \mathrm{~min}$ at each temperature, which were started after a few minutes' equilibration. The heating and temperature regulation procedure was to adjust the laser power, while monitoring the sample temperature using a pyrometer.

Microscopy. The in situ STEM experiments were performed at the ORNL Center for Nanophase Materials Sciences using a Protochips Aduro in situ heating holder and a Hitachi HF3300 S/TEM operating at $300 \mathrm{kV}$. The HEA samples were prepared by cutting a small piece ( $\sim 5 \mathrm{~mm}$ cube) from a bulk ingot using standing polishing. Thin, electron transparent specimens were then prepared using a Hitachi NB5000 focused ion beam (FIB) technique. The FIB-extracted specimen was then mounted to a resistive-heating chip, and placed in the S/TEM for examination at a series of temperatures, starting with ambient conditions. STEM images were collected at controlled elevated temperatures. The EDS data were collected using a Bruker silicon drift EDS detector after quenching to room temperature. Thus, the EDS measurements were not conducted at high temperatures. However, all STEM images were collected in situ at the reported temperatures. Note that the EDS and STEM images (Fig. 6) match well, indicating that no significant microstructural changes occurred due to temperature quenching between measurements.

Monte Carlo simulations. Monte Carlo simulations use random numbers to generate events, such as the jumping of atoms between lattice sites, which are accepted or rejected according to probabilities based upon physical models ${ }^{44}$. Here the mixing behavior of an HEA is simulated by populating a truncated lattice with multiple elements (two to six different elements), initially in a random configuration, or, alternately, in a sequence where each element fills a contiguous block of sites, representing an "unmixed" state. The simulation proceeds by allowing randomly selected atom pairs to exchange positions, or jump. The jumps are accepted or rejected according to a Boltzmann probability, $P$, given by

$$
P=\exp \frac{-\Delta H}{k_{\mathrm{B}} T},
$$

where $\Delta H$ is the enthalpy change caused by the jump, $k_{\mathrm{B}}$ is the Boltzmann constant, and $T$ is the temperature.

Each atom on the B2 lattice has eight nearest neighbors, and $\Delta H$ may be approximated, using the same binary interaction parameters, $v_{i j}$, as in the BraggWilliams model ${ }^{1}$. In contrast to the Bragg-Williams model, however, the Monte Carlo simulation evaluates $\mathrm{v}_{i j}$ for discrete atom pairs, throughout the lattice. Furthermore, the long-range order parameters, $\eta_{i}$, which are the required inputs for solving the Bragg-Williams expressions, may be extracted from the Monte Carlo simulations, without any prior knowledge of the ordering trends.

Consider two lattice sites, $L$ and $M$, which are occupied by elements of types, $l$ and $m$, respectively, and are surrounded by nearest-neighbor atoms of types, $l(k)$ and $m(k)$, respectively, where $k=1-8$ for the BCC structure. If the atoms on sites, $L$ and $M$, jump and trade places, then the $\Delta H$ used in the Boltzmann probability of Eq. (3) may be written as

$$
\Delta H_{\text {jump }}=\sum_{k=1}^{8}\left[\left(v_{l, m(k)}+v_{m, l(k)}\right)-\left(v_{l, l(k)}+v_{m, m(k)}\right)\right]
$$

The jump is always accepted, when $\Delta H_{\text {jump }}$ is negative, but accepted only by "chance" when $\Delta H_{\text {jump }}$ is positive, according to the probability, $P$, given by Eq. (3). As the Monte Carlo simulation is run for thousands of jumps, the sum of the $\Delta H_{\text {jump }}$ for all accepted jumps asymptotically approaches an equilibrium value, as the atoms reach an equilibrium configuration.

The long-range order parameters, $\eta_{i}$, were extracted from the simulation simply by comparing the elemental populations on each sublattice. In particular,

$$
\eta_{i}(\text { simulation })=\frac{N_{i \alpha}-N_{i \mathrm{Av}}}{N_{i \mathrm{Av}}}
$$

where $N_{i \alpha}$ is the number of type- $i$ atoms on the a sublattice, and $N_{i \mathrm{Av}}$ is the average number of type- $i$ atoms on each sublattice. Thus, the Monte Carlo simulation may inform the choice of $\eta_{i}$, used in the above described Bragg-Williams calculations. 
Differential scanning calorimetry. The solidification temperature and possible solid-solid phase transition temperature of the $\mathrm{AlCoCrNiFe}(x=1)$ equiatomic HEA were measured using a NETZSCH $404 \mathrm{C}$ differential scanning calorimeter. The measurement was run at a constant cool rate of $20 \mathrm{C} / \mathrm{min}$ from 1450 to $250^{\circ}$ $\mathrm{C}$ under a flowing argon atmosphere. Results are shown in Supplementary Figure 3.

Code availability. The Metropolis Monte Carlo used in this study was developed by L.J.S., for use within MATLAB. This code is available on request from Santodonato (lsantod1@vols.utk.edu) or the corresponding author J.R.M. (morrisj@ornl. gov).

\section{Data availability}

All figures have associated raw data, available on request from the corresponding author, J.R.M. (morrisj@ornl.gov), or from the first author, L.J.S. (lsantod1@vols. utk.edu).

Received: 10 October 2016 Accepted: 12 September 2018

Published online: 30 October 2018

\section{References}

1. Bragg, W. L. \& Williams, E. J. The effect of thermal agitation on atomic arrangement in alloys. Proc. R. Soc. Lond. Ser. A 145, 699-730 (1934).

2. Yeh, J. W. et al. Nanostructured high-entropy alloys with multiple principal elements: Novel alloy design concepts and outcomes. Adv. Eng. Mater. 6, 299-303 (2004).

3. Cantor, B., Chang, I. T. H., Knight, P. \& Vincent, A. J. B. Microstructural development in equiatomic multicomponent alloys. Mat. Sci. Eng. A 375, 213-218 (2004).

4. Miracle, D. B. \& Senkov, O. N. A critical review of high entropy alloys and related concepts. Acta Mater. 122, 448-511 (2017).

5. Gao, M. C., Yeh, J.-W., Liaw, P. K. \& Zhang, Y. High-Entropy Alloys (Springer International, Cham, 2016)

6. Gludovatz, B. et al. A fracture-resistant high-entropy alloy for cryogenic applications. Science 345, 1153-1158 (2014).

7. Troparevsky, M. C., Morris, J. R., Kent, P. R. C., Lupini, A. R. \& Stocks, G. M. Criteria for predicting the formation of single-phase high-entropy alloys. Phys. Rev. X 5, 011041 (2015).

8. Senkov, O. N., Miller, J. D., Miracle, D. B. \& Woodward, C. Accelerated exploration of multi-principal element alloys with solid solution phases. Nat. Commun. 6, 6529 (2015)

9. Zhang, Y. et al. Microstructures and properties of high-entropy alloys. Prog. Mater. Sci. 61, 1-93 (2013)

10. Santodonato, L. J. et al. Deviation from high-entropy configurations in the atomic distributions of a multi-principal-element alloy. Nat. Commun. 6, 5964 (2015).

11. Zhang, Z. et al. Nanoscale origins of the damage tolerance of the high-entropy alloy CrMnFeCoNi. Nat. Commun. 6, 10143 (2015).

12. Rost, C. M. et al. Entropy-stabilized oxides. Nat. Commun. 6, 8485 (2015).

13. Jin, K. et al. Tailoring the physical properties of Ni-based single-phase equiatomic alloys by modifying the chemical complexity. Sci. Rep. 6, 20159 (2016).

14. Zou, Y., Ma, H. \& Spolenak, R. Ultrastrong ductile and stable high-entropy alloys at small scales. Nat. Commun. 6, 7748 (2015).

15. Li, Z., Pradeep, K. G., Deng, Y., Raabe, D. \& Tasan, C. C. Metastable highentropy dual-phase alloys overcome the strength-ductility trade-off. Nature 534, 227-230 (2016).

16. Troparevsky, M. C. et al. Beyond atomic sizes and Hume-Rothery rules: understanding and predicting high-entropy alloys. JOM 67, 2350-2363 (2015).

17. Tong, C. J. et al. Microstructure characterization of $\mathrm{Al}_{\mathrm{x}} \mathrm{CoCrCuFeNi}$ highentropy alloy system with multiprincipal elements. Mat. Trans. A 36A, 881-893 (2005).

18. Tong, C. J. et al. Mechanical performance of the $\mathrm{A}_{\mathrm{lx}} \mathrm{CoCrCuFeNi}$ high-entropy alloy system with multiprincipal elements. Mat. Trans. A 36A, 1263-1271 (2005).

19. Chen, H. Y. et al. Effect of the substitution of Co by $\mathrm{Mn}$ in $\mathrm{Al}-\mathrm{Cr}-\mathrm{Cu}-\mathrm{Fe}-\mathrm{Co}-$ Ni high-entropy alloys. Ann. Chim. Sci. Mat. 31, 685-698 (2006).

20. Tsai, C. W., Tsai, M. H., Yeh, J. W. \& Yang, C. C. Effect of temperature on mechanical properties of $\mathrm{Al}_{0.5} \mathrm{CoCrCuFeNi}$ wrought alloy. J. Alloy. Compd. 490, 160-165 (2010).

21. Singh, S., Wanderka, N., Murty, B. S., Glatzel, U. \& Banhart, J. Decomposition in multi-component $\mathrm{AlCoCrCuFeNi}$ high-entropy alloy. Acta Mater. 59, 182-190 (2011).
22. Zhang, C. et al. Understanding phase stability of Al-Co-Cr-Fe-Ni high entropy alloys. Mater. Des. 109, 425-433 (2016).

23. Butler, T. M. \& Weaver, M. L. Investigation of the phase stabilities in AlNiCoCrFe high entropy alloys. J. Alloy. Compd. 691, 119-129 (2017).

24. Rao, J. C. et al. Secondary phases in AlxCoCrFeNi high-entropy alloys: An insitu TEM heating study and thermodynamic appraisal. Acta Mater. 131, 206-220 (2017).

25. Wang, Y. P., Li, B. S. \& Fu, H. Z. Solid solution or intermetallics in a highentropy alloy. Adv. Eng. Mater. 11, 641-644 (2009).

26. Welk, B. A. et al. Nature of the interfaces between the constituent phases in the high entropy alloy CoCrCuFeNiAl. Ultramicroscopy 134, 193-199 (2013).

27. Manzoni, A., Daoud, H., Völkl, R., Glatzel, U. \& Wanderka, N. Phase separation in equiatomic $\mathrm{AlCoCrFeNi}$ high-entropy alloy. Ultramicroscopy 132, 212-215 (2013).

28. Kao, Y. F., Chen, T. J., Chen, S. K. \& Yeh, J. W. Microstructure and mechanical property of as-cast, -homogenized, and -deformed $\mathrm{Al}(\mathrm{x})$ CoCrFeNi $(0<=\mathrm{x}<=2)$ high-entropy alloys. J. Alloy. Compd. 488, 57-64 (2009).

29. Gao, M. C. et al. Thermodynamics of concentrated solid solution alloys. Curr Opin. Solid State Mater. Sci. 21, 238-251 (2017).

30. Derimow, N., Santodonato, L., Mills, R. \& Abbaschian, R. In-situ imaging of liquid phase separation in molten alloys using cold neutrons. J. Imaging 4, 5 (2018).

31. Munitz, A., Kaufman, M. J. \& Abbaschian, R. Liquid phase separation in transition element high entropy alloys. Intermetallics 86, 59-72 (2017)

32. Huhn, W. P. \& Widom, M. Prediction of A2 to B2 phase transition in the high-entropy alloy Mo-Nb-Ta-W. JOM 65, 1772-1779 (2013).

33. Widom, M., Huhn, W. P., Maiti, S. \& Steurer, W. Hybrid Monte Carlo/ molecular dynamics simulation of a refractory metal high entropy alloy. Mat. Trans. A 45, 196-200 (2014).

34. Sun, X. et al. Phase selection rule for Al-doped $\mathrm{CrMnFeCoNi}$ high-entropy alloys from first-principles. Acta Mater. 140, 366-374 (2017).

35. Weber, J. K. R., Felten, J. J., Cho, B. \& Nordine, P. C. Glass fibres of pure and erbium- or neodymium-doped yttria-alumina compositions. Nature 393 , 769-771 (1998)

36. Weber, J. K. R. et al. Measurements of liquid and glass structures using aerodynamic levitation and in-situ high energy $\mathrm{X}$-ray and neutron scattering. J. Non-Cryst. Solids 383, 49-51 (2014).

37. Fultz, B. Vibrational thermodynamics of materials. Prog. Mater. Sci. 55, 247-352 (2010)

38. Fultz, B. et al. Phonon modes and vibrational entropy of mixing in $\mathrm{Fe}-\mathrm{Cr}$. Phys. Rev. B 52, 3280-3285 (1995).

39. Swan-Wood, T. L., Delaire, O. \& Fultz, B. Vibrational entropy of spinodal decomposition in FeCr. Phys. Rev. B 72, 024305 (2005).

40. Körmann, F. \& Sluiter, M. Interplay between lattice distortions, vibrations and phase stability in NbMoTaW high entropy alloys. Entropy 18, 403 (2016).

41. Lovesey, S. W. Theory of Neutron Scattering from Condensed Matter (Clarendon Press, Oxford, 1984).

42. Pynn, R. Neutron scattering: a primer. Los Alamos. Sci. 19, 1-31 (1990).

43. Neuefeind, J., Feygenson, M., Carruth, J., Hoffmann, R. \& Chipley, K. K. The nanoscale ordered materials diffractometer NOMAD at the Spallation Neutron Source SNS. Nucl. Instrum. Methods Phys. Res. B 287, 68-75 (2012).

44. Landau, D. P. \& Binder, K. A Guide to Monte Carlo Simulations in Statistical Physics 4th edn (Cambridge University Press, Cambridge, 2014).

\section{Acknowledgements}

J.R.M. and H.B. acknowledge support from the U.S. Department of Energy (USDOE), Office of Science, Basic Energy Sciences, Materials Science and Engineering Division (theory and calorimetry experiment). L.J.S. (neutron scattering and MC simulations) acknowledges the support and resources at the High Flux Isotope Reactor and Spallation Neutron Source, USDOE Office of Science user facilities operated by the Oak Ridge National Laboratory. In situ microscopy was conducted at the Center for Nanophase Materials Sciences, which is a DOE Office of Science User Facility. Chih-Hsiang (Sean) Kuo and Chan-ho Lee helped conduct preliminary microscopy work. P.K.L. (discussion and interpretation of results) acknowledges the support from the US Army Research Office Project (W911NF-13-1-0438), with Dr. M.P. Bakas, Dr. D.M. Stepp, and S.N. Mathaudhu as program managers. P.K.L. thanks the support of the National Science Foundation, Grant DMR-1611180, under program directors of Dr. G. Shiflet and Dr. D. Farkas.

\section{Author contributions}

J.R.M. initiated the research concept and supplied the initial Monte Carlo code. L.J.S extended the Monte Carlo code and ran the present simulations. L.J.S. conducted the neutron scattering experiments. L.J.S. and R.R.U. conducted the in situ microscopy experiments. H.B. performed the calorimetry work. L.J.S. and J.R.M. interpreted the 
simulations and wrote the manuscript, with significant input from all other authors. J.R.M. and P.K.L. supervised the project.

\section{Additional information}

Supplementary Information accompanies this paper at https://doi.org/10.1038/s41467018-06757-2.

Competing interests: The authors declare no competing interests.

Reprints and permission information is available online at http://npg.nature.com/ reprintsandpermissions/

Publisher's note: Springer Nature remains neutral with regard to jurisdictional claims in published maps and institutional affiliations. (c) (i) Open Access This article is licensed under a Creative Commons Attribution 4.0 International License, which permits use, sharing, adaptation, distribution and reproduction in any medium or format, as long as you give appropriate credit to the original author(s) and the source, provide a link to the Creative Commons license, and indicate if changes were made. The images or other third party material in this article are included in the article's Creative Commons license, unless indicated otherwise in a credit line to the material. If material is not included in the article's Creative Commons license and your intended use is not permitted by statutory regulation or exceeds the permitted use, you will need to obtain permission directly from the copyright holder. To view a copy of this license, visit http://creativecommons.org/ licenses/by/4.0/.

(C) The Author(s) 2018 